\section{REVISTA}

MEXICANA DE

ECONOMÍA Y

FINANZAS

REMEF

(THE MEXICAN JOURNAL OF

ECONOMICS AND FINANCE)
Revista Mexicana de Economía y Finanzas Nueva Época

Volumen 15 Número 2, Abril - Junio 2020, pp. 277-293

DOI: https://doi.org/10.21919/remef.v15i2.485

(Recibido: 15/abril/2019, aceptado: 1/agosto/2019)

\title{
Determinantes financieras de la Sustentabilidad Corporativa de Empresas que cotizan en el IPC Sustentable de la BMV
}

\author{
Nora Gavira-Durón ${ }^{1}$ \\ Universidad de las Américas Puebla, México \\ Dolores Guadalupe Martínez Peña ${ }^{2}$ \\ Universidad Michoacana de San Nicolás de Hidalgo, México \\ Irma Cristina Espitia Moreno \\ Universidad Michoacana de San Nicolás de Hidalgo, México
}

\section{Resumen}

El objetivo de la investigación es determinar si los indicadores: retorno sobre activos (ROA), Quick-ratio (quickr), tamaño total de activos (tactivos), margen de beneficios antes de intereses, impuestos, depreciación y amortización (ebitdam), z-score (zaltman); de las empresas del IPC sustentable de la BMV, impactan a los índices: ambiental (IA), responsabilidad social (IS), gobierno corporativo (IGC) y su índice general: Desempeño Sustentable Corporativo (DSC). Con un modelo de panel con Mínimos Cuadrados Ordinarios Agrupados y efectos fijos en secciones cruzadas y periodos, con apoyo de EVIEWS 9. Los resultados muestran que las variables significativas fueron tactivos y zaltman, que existe una relación inversa entre ellas y DSC, IA, IS e IGC. Se recomienda analizar todas las empresas del índice, cuando se cuente con mayor historial de datos y aplicar otros modelos estadísticos. Las limitaciones son el bajo número de observaciones y la utilización de un sólo modelo, esto implica resultados no concluyentes; sin embargo, es relevante ya que no se había estudiado el caso mexicano. Se concluye que las empresas tienen un costo extra por formar parte del índice.

Clasificación JEL: C02, C13, C51, Q50, Q56

Palabras claves: IPC sustentable, desempeño ambiental, responsabilidad social, gobierno corporativo, desempeño financiero

\section{Financial Determinants of the Corporate Sustainability of Companies listed in the Sustainable IPC of the BMV}

\section{Abstract}

The objective of the present research is to observe if the following five indicators from companies listed on the Mexican Sustainability Index (IPCS): return on assests (ROA), Quick-ratio (quickr), total assests (tactivos). Earnings Before Interest, Tax, Depreciation, Amortization Margin (ebitdam) and z-score (zaltman) have an impact on: environment index (IA), social responsibility index (IS), corporate governance (IGC) and

\footnotetext{
${ }^{1}$ Mail: nora.gavira@udlap.mx

${ }^{2}$ Mail: martinezpdolores@gmail.com

* Sin fuente de financiamiento declarada para el desarrollo de la investigación
} 


\section{Abstract}

sustainability index (DSC), through panel data model using least squared, fixed effects in crossed sections and periods using EVIEWS 9. The findings show that tactivos and zaltman were the significative variables, indicating a negative correlation between them and DSC, IA, IS e IGC. The findings suggest that all companies listed should be assessed in the future when more data is available. The limitations of this study are: the small size of the sample and just a single model in consequence the results are inconclusive nevertheless the work is relevant since these variables have not been studied in the Mexican scenario. Another conclusion is that the companies listed have extra costs derived from pertaining to the present index.

JEL Classification: C02, C13, C51, Q50, Q56

Keywords: Sustainable IPC, environmental performance, social responsibility, corporate governance, financial performance

\section{Introducción}

Los mercados son cada vez más competitivos y la velocidad de este cambio está generando a las compañías una gran presión, no sólo por ser exitosas, sino que además deben planear y realizar las estrategias necesarias para mantener su éxito en el futuro, por lo que, la sustentabilidad corporativa ha sido la gran protagonista durante los últimos años. Empresas, inversionistas y consumidores, han puesto su atención en esta temática; todo lo que implica y se deriva de ella, Ameer \& Othman (2012) y Lourenço, Branco, Curto, \& Eugénio (2012). Por lo tanto, se espera que las empresas vayan más allá del enfoque financiero a corto y largo plazo, y que tiendan a una sustentabilidad económica, ambiental y social Haffar \& Searcy (2017).

La sustentabilidad corporativa ha tomado el papel del nuevo paradigma en la administración de las empresas y representa una alternativa al modelo de crecimiento tradicional y de maximización de la rentabilidad Wilson (2003). Para Székely \& Knirsch (2005), muchas empresas han iniciado una variedad de iniciativas de desarrollo sustentable para atender las demandas y expectativas de la sociedad. Aun cuando un gran número de analistas aseguran que estas iniciativas contribuyen para hacer negocios más rentables, existen todavía muchos gerentes que siguen sin aceptar la validez de este argumento, la causa de esta discrepancia entre analistas y gerentes podría ser que la mayoría de las iniciativas de desarrollo sustentable se han desarrollado sin la participación de la actividad comercial. Por lo tanto, estas iniciativas no están directamente vinculadas a la estrategia empresarial.

En México, la Bolsa Mexicana de Valores Indices (2019) lanzó al mercado el Índice de Precios y Cotizaciones Sustentable (S\&P/BMV IPC Sustentable), el 8 de diciembre de 2011 para medir el rendimiento de aquellas empresas listadas en IPC que presentan los mejores niveles sustentables. Por lo anterior, la hipótesis a contrastar es si a mayor desempeño financiero de las empresas que cotizan en el IPC Sustentable o índice verde de la Bolsa Mexicana de Valores (BMV); entonces, se tiene un mayor Desempeño Sustentable Corporativo (DSC), medido también por sus componentes: Medio Ambiente (IA), Responsabilidad Social (IS) y Gobierno Corporativo (IGC).

La calificación de sustentabilidad para cada empresa que cotiza en la BMV, se realiza por el Centro de Excelencia en Gobierno Corporativo (CEGC) de la Universidad Anáhuac México Sur, se establece en función de los resultados de la evaluación de las prácticas a largo plazo en materia ambiental, social y de aquellas que sean a fin a las tendencias de sustentabilidad de la industria correspondiente a la que pertenezcan (S\&P Dow Jones Indices, 2018). Se otorga una calificación conjunta para el DSC de acuerdo al promedio obtenido en relación a sus componentes (IA, IS e IGC). Las evaluaciones de estos componentes se obtuvieron de la página web de Yahoo Finanzas. Las series de indicadores financieros para las empresas analizadas, se obtuvieron de 
la plataforma Standard \& Poor's Capital IQ.

La metodología a aplicar es un modelo de panel con Mínimos Cuadrados Ordinarios Agrupados y efectos fijos en secciones cruzadas y periodos, para DSC y cada uno de sus subíndices: IA, IS, e IGC; considerando como variables independientes a los indicadores financieros trimestrales (ROA, quickr, tactivos, ebitdam y zaltman) de 2015 a 2018, de una muestra de 13 empresas que cotizan en el IPC Sustentable de la BMV. Para el análisis, se utiliza el paquete estadístico EVIEWS 9.

En la sección 2 se presenta la revisión de la literatura y los principales trabajos que se han realizado para determinar el impacto de los indicadores financieros en la sustentabilidad de las empresas; en la sección 3 se analiza el desarrollo de los índices bursátiles sustentables en todo el mundo y en particular en México; en la sección 4 se detalla la muestra a analizar y los modelos de panel de datos que se aplicarán; en la sección 5 se presentan los resultados y se contrastan con los obtenidos en estudios previos; en la sección 6 se presentan las conclusiones, recomendaciones, limitaciones e implicaciones.

\section{Revisión de la literatura}

Marichalar de Corral (2014), identifica tres tipos de criterios o modelos de evaluación de la sustentabilidad corporativa: En primer lugar, los sistemas de gestión de la ética corporativa relacionados al cumplimiento del código ético de las empresas y elaborado en función de las características de las mismas, conocido como "Compliance". En segundo lugar, se encuentran los índices bursátiles sustentables que integran sólo a las empresas que cumplen con criterios específicos establecidos por las bolsas de valores donde cotizan y por último se identifican los rankings elaborados por diferentes instituciones o empresas de consultoría contables o financieras globales que toman diferentes criterios para la elaboración de sus respectivos índices.

Fue hasta 1980 cuando el concepto de Responsabilidad Social Corporativa (RSC) evolucionó en términos de la teoría de los stakeholders (grupos de interés o de las partes interesadas) desarrollada a partir del trabajo realizado por R. Edward Freeman en 1984, que sentó la base para comprender la relación entre la RSC y el desarrollo sustentable durante los años noventa. De acuerdo con Goodpaster, Rodbourne, Hernke, \& Betzler (2010), las definiciones de RSC han surgido tanto de profesionales en los negocios como de académicos, pero de manera casi y totalmente independiente.

Por lo que Wang, Tong, Takeuchi, \& George (2016) establecen que para comprender el entorno institucional de la RSC es importante aceptar que es un fenómeno social; que no es independiente del contexto institucional de la empresa porque examina las actividades sociales corporativas y su influencia sobre las empresas y todas sus partes interesadas.

Para Hamidu, Haron, \& Amran (2015) la Responsabilidad Social Corporativa tiene varias definiciones debido a la variación del propósito de la misma, entre los principales se encuentran el cumplimiento de las obligaciones para con la comunidad que rodea a la empresa, firma, organización o corporación, la construcción de la reputación corporativa y el desarrollo social. En síntesis y de acuerdo con Bhaduri \& Selarka (2016), en términos simples, la RSC puede entenderse a partir de las palabras que la conforman: Çorporativo"que abarca el amplio espectro de negocios, "Social"que se refiere a la comunidad local con la que interactúan y finalmente Responsabilidades"que son intrínsecas a ambos lados de estas relaciones.

\subsection{Análisis de estudios previos}

Por otra parte, existen diversas investigaciones que analizan la relación existente entre la sustentabilidad corporativa de las empresas y su rentabilidad financiera, como se muestra en la Tabla 1. 
Tabla 1. Investigaciones sobre la relación entre Sustentabilidad de las empresas y su desempeño financiero

\begin{tabular}{|c|c|c|c|}
\hline Estudio y país & Medida de SC & $\begin{array}{l}\text { Medida de rendimiento } \\
\text { financiero }\end{array}$ & $\begin{array}{c}\text { Resultado de la } \\
\text { relación } \\
\text { encontrada }\end{array}$ \\
\hline $\begin{array}{l}\text { Jones }(2005)- \\
\text { Australia }\end{array}$ & $\begin{array}{c}\text { Puntaje del Índice de Informes de } \\
\text { Sustentabilidad GRI (Global } \\
\text { Reporting Initiative) }\end{array}$ & $\begin{array}{l}\text { Devoluciones ajustadas al } \\
\text { mercado; otras razones } \\
\text { financieras; y puntajes de } \\
\text { probabilidad de dificultad } \\
\text { financiera }\end{array}$ & $\begin{array}{c}\text { Resultados mixtos } \\
\text { con diferentes medidas } \\
\text { de rendimiento de la } \\
\text { empresa }\end{array}$ \\
\hline $\begin{array}{l}\text { Van de Velde, } \\
\text { Vermeir, \& } \\
\text { Corten }(2005)- \\
\text { Europe }\end{array}$ & $\begin{array}{l}\text { Puntuaciones de sustentabilidad } \\
\text { de Vigeo: recursos humanos, } \\
\text { medio ambiente, clientes y } \\
\text { proveedores, comunidad y } \\
\text { sociedad, y gobierno corporativo }\end{array}$ & $\begin{array}{l}\text { Promedio de devoluciones } \\
\text { mensuales en cartera }\end{array}$ & $\begin{array}{c}\text { Positivo, pero no } \\
\text { significativo }\end{array}$ \\
\hline $\begin{array}{l}\text { Brammer, } \\
\text { Brooks, \& } \\
\text { Pavelin (2006) - UK }\end{array}$ & $\begin{array}{c}\text { Puntaje de Sustentabilidad } \\
\text { Compuesto / Agregado de la base } \\
\text { de datos EIRIS } \\
\text { Rendimientos de las acciones } \\
\text { Negativo }\end{array}$ & Rendimiento de las acciones & Negativo \\
\hline $\begin{array}{l}\text { Moneva, } \\
\text { Archel, \& } \\
\text { Correa }(2006)- \\
\text { Europe }\end{array}$ & $\begin{array}{l}\text { Divulgaciones en el Informe de } \\
\text { Sustentabilidad GRI }\end{array}$ & Share Price Returns & No significativa \\
\hline $\begin{array}{l}\text { Hindley \& } \\
\text { Buys (2012)- } \\
\text { South Africa }\end{array}$ & $\begin{array}{l}\text { Presentación de los informes de } \\
\text { sustentabilidad GRI }\end{array}$ & $\begin{array}{c}\text { ROA, ROE, EVA \& MVA } \\
\text { (Market Value Added) }\end{array}$ & $\begin{array}{c}\text { Ligeramente positiva, } \\
\text { pero no significativa }\end{array}$ \\
\hline $\begin{array}{l}\text { Dhaliwal, } \\
\text { Zhen Li, Tsang, \& } \\
\text { Yang (2011) - US }\end{array}$ & MSCI KLD Ratings & Costo del capital social & Negativa \\
\hline $\begin{array}{l}\text { Ameer \& } \\
\text { Othman (2012) } \\
\text { países desarrollados }\end{array}$ & $\begin{array}{l}\text { Puntuaciones en } 4 \text { índices: medio } \\
\text { ambiente, diversidad, comunidad } \\
\text { y ética }\end{array}$ & $\begin{array}{l}\text { Crecimiento de ingresos } \\
\text { de ventas (SRG), ROA, } \\
\text { PBT y CFO }\end{array}$ & $\begin{array}{l}\text { Relación positiva y } \\
\text { bidireccional }\end{array}$ \\
\hline $\begin{array}{l}\text { Bayoud \& } \\
\text { Kavanagh (2012 )- } \\
\text { Libya }\end{array}$ & $\begin{array}{l}\text { Divulgación de la participación } \\
\text { ambiental, del consumidor, de la } \\
\text { comunidad, Rendimiento de los } \\
\text { empleados }\end{array}$ & ROA, Ingresos, ROI & Positiva \\
\hline $\begin{array}{l}\text { Eccles, Krzus, } \\
\text { Rogers, \& Serafeim } \\
(2012) \text { - US }\end{array}$ & $\begin{array}{c}\text { Puntuaciones de divulgación de } \\
\text { ESG de la base de datos Asset4, } \\
\text { Bloomberg y SAM }\end{array}$ & $\begin{array}{l}\text { Rendimientos de las } \\
\text { acciones, ROA, ROE }\end{array}$ & Positiva \\
\hline $\begin{array}{l}\text { Burhan \& } \\
\text { Rahmanti (2012) - } \\
\text { Indonesia }\end{array}$ & $\begin{array}{c}\text { Puntaje del índice de divulgación } \\
\text { basado en GRI }\end{array}$ & ROA & Positiva \\
\hline $\begin{array}{l}\text { Venanzi (2013) - } \\
\text { Europe }\end{array}$ & $\begin{array}{c}\text { Clasificaciones sociales en } \\
\text { comunidad, gobierno corporativo, } \\
\text { clientes, empleados, medio } \\
\text { ambiente, proveedores, ética } \\
\text { comercial y controversias. }\end{array}$ & ROE, ROA, ROS. & No significativa \\
\hline $\begin{array}{l}\text { Motwani \& } \\
\text { Pandya (2016)- } \\
\text { India }\end{array}$ & $\begin{array}{c}\text { OSR- Calificación general de } \\
\text { sostenibilidad } \\
\text { COM- Evaluación del Desempeño } \\
\text { de la Comunidad } \\
\text { EMP- Calificación de rendimiento } \\
\text { de los empleados } \\
\text { ENV- Calificación de desempeño } \\
\text { ambiental } \\
\text { GOV- Calificación de rendimiento } \\
\text { de gobierno tomadas de CSRHub }\end{array}$ & $\begin{array}{l}\text { ROA, ROE, ROCE, } \\
\text { PBT \& GTA }\end{array}$ & $\begin{array}{c}\text { Positiva y significativa } \\
\text { hasta cierto punto. }\end{array}$ \\
\hline
\end{tabular}

Fuente: Elaboración propia con información de Aggarwal (2013) 
Se puede observar que no se han desarrollado investigaciones para el caso de los países de centro y Sudamérica; en particular para México. Por lo anterior, el objetivo de la presente investigación se centra en determinar si los indicadores financieros: retorno sobre los activos (ROA), Quick ratio (quickr), tamaño total de los activos (tactivos), margen de beneficios antes de intereses, impuestos, depreciación y amortización (ebitdam), y ratio del puntaje de la z-score (zaltman), de las empresas que forman el IPC sustentable de la BMV impactan al índice ambiental (IA), índice de responsabilidad social (IS), índice de gobierno corporativo (IGC) y en general al Desempeño Sustentable Corporativo (DSC).

\section{3. Índices Bursátiles Sustentables}

El primer índice bursátil sustentable en el mundo fue el "Domini 400 Social Index" lanzado en mayo de 1990 por Kinder, Lydenberg, Domini \& Co, mismo que hoy en día conocemos como el MSCI KLD 400 Social Index, MSCI (2019); fue creado en Estados Unidos de América y considera a las empresas con las mejores clasificaciones en los rubros ambientales, sociales y de gobierno. En 1999, S\&P Dow Jones Indicesen conjunto con RobecoSAM, crean la familia del Dow Jones Sustainability Index (DJSI), ROBECOSAM (2019), formado por un conjunto de índices de sostenibilidad de las empresas con alto desempeño económico, social y ambiental; los índices representan un punto de referencia para los inversionistas que consideran a las prácticas comerciales sostenibles como fundamentales para generar valor a largo plazo. En 2001 se genera en Londres, el Financial Times Stock Exchange, FTSERussell (2019) que representa una serie de índices bursátiles de la Bolsa de Londres. La serie de índices FTSE4Good, Russell (2019), la cual, está diseñada para medir el desempeño de las empresas de todo el mundo que comparten prácticas sólidas en materia de Medio Ambiente, Social y Gobernabilidad. Los índices de sostenibilidad de Ethibel (ESI) se establecieron en Bélgica, en junio de 2002, ETHIBEL (2019), y desde entonces se consideran como un punto de referencia para la sostenibilidad corporativa y social; así como de la inversión responsable. En América Latina se genera en Brasil el Índice de Sustentabilidad Empresarial (ISE B3) en el año 2005, BOVESPA (2019), con la intención de crear un entorno de inversión compatible con el desarrollo sostenible. En 2007, en Estados Unidos de américa, se crea el MSCI ACWI ESG Leaders Index, INC (2019), el cual contiene empresas de alto nivel en los rubros: ambiental, desempeño social y de gobierno, considerando sus pares del mismo sector.

El Dow Jones Sustainability Asia Pacific Index (DJSI Asia Pacific) se genera con el puntaje otorgado a cada empresa, de acuerdo con la metodología de evaluación de sostenibilidad de Robeco, CLP (2019), CLP es un componente de los índices IDJSI Asia Pacific y Dow Jones Sustainability Asia Pacific 40 (DJSI Asia Pacific 40) desde el lanzamiento de ambos índices en 2009. Como parte de la familia de los índices del Dow Jones Sustainability se genera el Dow Jones Sustainability Chile en 2015, compuesto por empresas líderes en sostenibilidad, considerando su puntaje de sostenibilidad, con base en factores ambientales, sociales y de buen gobierno corporativo de largo plazo. Posteriormente, en el tercer trimestre de 2017 se creó el Dow Jones Sustainability MILA Pacific Alliance (DJSI MILA Pacific, Alliance), S\&P D. J. (2019), primer índice regional con el objetivo de medir el desempeño sustentable de empresas chilenas, colombianas, mexicanas y peruanas; en la emisión 2017 se encontraban $12^{3}$ empresas mexicanas que cotizan en la BMV, para 2018 se integró Alsea SAB de CV; con lo que 13, EY (2019) de las 42 organizaciones que forman el índice son mexicanas.

Más recientemente, en mayo de 2018 se desarrolla en Argentina, BYMA (2019), el Índice de Sustentabilidad BYMA, el cual considera para su integración, el factor ambiental, social y de gobierno corporativo.

\footnotetext{
${ }^{3}$ Alsea, Axtel, Cemex, Coca-Cola FEMSA, Fibra Uno, FEMSA, Grup Televisa, Grupo Financiero Banorte, Grupo México, Grupo Rotoplas, Proligis property México, Qualitas Controladora y OMA.
} 


\section{1. Índice de Precios y Cotizaciones Sustentable de la Bolsa Mexicana de Va- lores}

En México, fue hasta el 8 de diciembre de 2011 cuando la Bolsa Mexicana de Valores, Indices (2019) lanzó al mercado el Índice de Precios y Cotizaciones Sustentable (S\&P/BMV IPC Sustentable), con el objetivo de medir el rendimiento de las empresas listadas con los mejores niveles sustentables, inicialmente fue constituido por las $23^{4}$ empresas mejor calificadas en desempeño ambiental, responsabilidad social y gobierno corporativo, al cierre de 2018 se incrementó a $30^{5}$. Este índice desde su origen ha buscado establecer el grado de adhesión a las mejores prácticas internacionales, en función al análisis de la información pública disponible que cada emisora divulga en sus reportes o sitios web.

La calificación se realiza analizando los principios, sistemas y reportes de cada una de las emisoras de acuerdo a los principios relevantes del Pacto Mundial, los Principios de Inversión de Inversión Responsable (UN-PRI), las recomendaciones de la Organización para la Cooperación y el Desarrollo Económico (OCDE) y la Corporación Financiera Internacional del Banco Mundial que se encarga a nivel mundial de la promoción del buen Gobierno Corporativo y por último las recomendaciones de la norma ISO 14000 para la Industria Limpia, Bolsa Mexicana de Valores (2014).

Para la selección de los componentes, todas las series accionarias del universo elegible requieren contar con una calificación de sustentabilidad realizada por el Centro de Excelencia en Gobierno Corporativo (CEGC) de la Universidad Anáhuac México Sur. Esta calificación de sustentabilidad para cada una de las empresas se establece en función de los resultados de la evaluación exhaustiva de las prácticas a largo plazo en materia ambiental, social y de aquellas que sean a fin a las tendencias de sustentabilidad de la industria correspondiente a la que pertenezcan, S\&P Dow Jones Indices (2018). El Centro de Excelencia en Gobierno Corporativo de la Universidad Anáhuac México Sur, otorga una calificación conjunta para el Desempeño Sustentable Corporativo (DSC), de cada compañía de acuerdo al promedio obtenido en relación a las siguientes componentes:

a) Medio Ambiente (IA)

b) Responsabilidad Social (IS)

c) Gobierno Corporativo (IGC)

Cada año, se realiza un promedio de los puntajes obtenidos en cada uno de los tres factores señalados (IA, IS E IGC) para emitir una calificación final (DSC) para las compañías evaluadas, que posteriormente serán ordenadas de forma descendente de acuerdo a sus calificaciones finales obtenidas. Una vez que han sido ordenadas de manera descendente, sólo aquellas empresas clasificadas en el $80 \%$ del DSC más alto, se toman en cuenta para el cálculo de la puntuación media de la muestra. Las empresas con un puntaje final de desempeño sustentable por debajo de este promedio se descartan del Universo de Selección del índice. Sin embargo, a las empresas con un puntaje final por arriba de este promedio se les realiza una revisión adicional.

\footnotetext{
${ }^{4}$ Alfa, América Móvil, Arca Continental, Cemex, Coca-Cola Femsa, Compartamos Banco, Controladora Comercial Mexicana, Corporación Geo, Desarrolladora Homex, Empresas ICA, Fomento Económico Mexicano, Grupo Aeroportuario del Centro Norte, Grupo Aeroportuario del Sureste, Grupo Bimbo, Grupo Financiero Banorte, Grupo México, Grupo Modelo, Industrias Peñoles, Kimberly-Clark de México, Mexichem, TV Azteca, Urbi Desarrollos Urbanos y Wal-Mart de México.

${ }_{5}^{5}$ Alfa, Alsea, AMX, Arca Continental, Axtel, SANMEX, Bolsa Mexicana de Valores, Cemex, Coca-Cola Femsa, Volaris, Vesta, Fomento Económico Mexicano, Genoma Lab, GENTERA, OMA, ASUR, Grupo Bimbo, Elektra, Grupo Financiero Banorte, Grupo Herdez, LALA, Grupo México, Grupo Televisa, Hoteles City Express, Industrias Peñoles, IENOVA, Kimberly-Clark de México, Walmex y Rassini.
} 


\section{Metodología}

Actualmente la principal discusión sobre la inversión socialmente responsable (SRI) está enfocada en analizar y establecer de forma clara que tipo de relación existe entre el desempeño social corporativo de las empresas (CSP) y el desempeño financiero corporativo (CFP). Lamentablemente, los resultados de los estudios que se han realizado hasta el momento no permiten definir con total certeza el tipo de relación que guardan estas variables y de hecho se han identificado algunas limitaciones importantes, Xiao, Faff, Gharghori, \& Lee (2013). Algunos de los estudios que se han realizado para determinar si los indicadores financieros de las empresas, determinan la sustentabilidad de las mismas y sus resultados, se muestran en la Tabla 2.

Tabla 2. Variables financieras más utilizadas como determinantes de la sustentabilidad corporativa de las

\begin{tabular}{|c|c|}
\hline Variable & Autores \\
\hline $\begin{array}{l}\text { Retorno sobre los } \\
\text { activos ROA }\end{array}$ & $\begin{array}{l}\text { Artiach, Lee, Nelson, \& Walker (2010), Reverte (2009), Aggarwal (2013), } \\
\text { Motwani \& Pandya (2016), Waddock \& Graves (1997), Nigro, Iannuzzi, } \\
\text { Cortese, \& Petracca (2015), Kocmanova, Simanaviciene, \& Docekalova (2015), } \\
\text { Docekalova, Kocmanová, \& Koleňák (2015), Soytas, Denizel, \& Usar (2017) }\end{array}$ \\
\hline $\begin{array}{l}\text { Retorno sobre las } \\
\text { acciones ROE }\end{array}$ & $\begin{array}{l}\text { Lourenço \& Branco (2013), Aggarwal (2013), Motwani \& Pandya (2016), } \\
\text { Waddock \& Graves (1997), Nigro, Iannuzzi, Cortese, \& Petracca (2015), } \\
\text { Kocmanova, Simanaviciene, \& Docekalova (2015), Docekalova, Kocmanová, \& } \\
\text { Koleňák (2015), Soytas, Denizel, \& Usar (2017) }\end{array}$ \\
\hline $\begin{array}{l}\text { Retorno sobre el capital } \\
\text { empleado ROCE }\end{array}$ & Aggarwal (2013), Motwani \& Pandya (2016) \\
\hline $\begin{array}{l}\text { Beneficio antes de } \\
\text { impuestos PBT }\end{array}$ & Aggarwal (2013), Motwani \& Pandya (2016) \\
\hline $\begin{array}{l}\text { El tamaño total de } \\
\text { activos o el crecimiento } \\
\text { en los activos }\end{array}$ & $\begin{array}{l}\text { Artiach, Lee, Nelson, \& Walker (2010), Reverte (2009), Lourenço \& Branco (2013), } \\
\text { Aggarwal (2013), Motwani \& Pandya (2016), Waddock \& Graves (1997), } \\
\text { Lourenço, Branco, Curto, \& Eugénio (2012), Nigro, Iannuzzi, Cortese, \& } \\
\text { Petracca (2015), Soytas, Denizel, \& Usar (2017) }\end{array}$ \\
\hline $\begin{array}{l}\text { Precio valor en libro } \\
\text { de las acciones PB }\end{array}$ & $\begin{array}{l}\text { Artiach, Lee, Nelson, \& Walker (2010), Lourenço \& Branco (2013), Lourenço, } \\
\text { Branco, Curto, \& Eugénio (2012) }\end{array}$ \\
\hline $\begin{array}{l}\text { Apalancamiento } \\
\quad \text { (leverage) }\end{array}$ & $\begin{array}{l}\text { Artiach, Lee, Nelson, \& Walker (2010), Reverte (2009), Lourenço \& Branco (2013), } \\
\text { Lourenço, Branco, Curto, \& Eugénio (2012) }\end{array}$ \\
\hline $\begin{array}{l}\text { Flujo de caja libre } \\
\text { FCF (free cash Flow) }\end{array}$ & Artiach, Lee, Nelson, \& Walker (2010), Lourenço, Branco, Curto, \& Eugénio (2012) \\
\hline $\begin{array}{l}\text { Concentración de la } \\
\text { propiedad de la empresa }\end{array}$ & Reverte (2009), Lourenço \& Branco (2013) \\
\hline
\end{tabular}

En el caso de nuestro estudio, se analizará si los indicadores de sustentabilidad: Desempeño Sustentable Corporativo (DSC) y sus tres dimensiones: Índice de Desempeño Ambiental (IA), Índice de Responsabilidad Social (IS) e Índice de Gobierno Corporativo (IGC), se ven impactados por los indicadores financieros: retorno sobre los activos (ROA), Quick ratio (quickr), tamaño total de los activos (tactivos), margen de beneficios antes de intereses, impuestos, depreciación y amortización (ebitdam), y ratio del puntaje de la z-score (zaltman).

Las evaluaciones de los componentes de DSC, IA, IS e IGC se obtuvieron de la página web de Yahoo Finanzas. Las evaluaciones que se ofrecen de estos criterios son realizadas por Sustainalytics, una empresa holandesa que evalúa la sustentabilidad de las empresas que cotizan en diferentes bolsas de valores en el mundo 
en función de su desempeño ambiental, responsabilidad social y gobierno corporativo o índices ESG por sus siglas en inglés (Environmental, social and governance). Para obtener la información sobre los indicadores financieros elegidos como determinantes de la sustentabilidad corporativa de las empresas mexicanas que cotizan en la BMV, se consultó la plataforma Standard \& Poors Capital IQ.

\subsection{Selección de la muestra}

$\mathrm{Al}$ cierre de 2018, 30 empresas cotizan en el IPC Sustentable de la BMV; sin embargo, para la presente investigación se seleccionó una muestra con 13 empresas emisoras, las cuales han sido las más constantes en el índice, en el periodo trimestral de análisis de 2015 a 2018; además de que fueron las que presentaron información pública sobre la evaluación de su desempeño sustentable. Las empresas seleccionadas se muestran en la Tabla 3.

Tabla 3. Empresas mexicanas seleccionadas

\begin{tabular}{|c|c|c|c|}
\hline Nombre de la empresa & Ticker & Nombre de la empresa & Ticker \\
\hline Alfa, S. A. B. de C. V. & (ALFAA.MX) & $\begin{array}{l}\text { América Móvil, } \\
\text { S.A.B. de C.V. }\end{array}$ & (AMXL.MX) \\
\hline $\begin{array}{l}\text { Arca Continental, } \\
\text { S. A. B. de C. V. }\end{array}$ & (AC.MX) & $\begin{array}{l}\text { CEMEX, S.A.B. } \\
\text { de C.V. }\end{array}$ & (CEMEXCPO.MX) \\
\hline $\begin{array}{l}\text { Coca-Cola FEMSA, } \\
\text { S.A.B. de C.V. }\end{array}$ & (KOFL.MX) & $\begin{array}{l}\text { Grupo Bimbo, } \\
\text { S.A.B. de C.V. }\end{array}$ & (BIMBOA.MX) \\
\hline $\begin{array}{l}\text { Fomento Económico } \\
\text { Mexicano, S.A.B. de C.V. } \\
\text { FEMSA UBD Units }\end{array}$ & (FEMSAUBD.MX) & $\begin{array}{l}\text { Wal-Mart de México, } \\
\text { S.A.B. de C.V. }\end{array}$ & (WALMEX.MX) \\
\hline $\begin{array}{l}\text { Grupo México, } \\
\text { S.A.B. de C.V. }\end{array}$ & (GMEXICOB.MX) & $\begin{array}{l}\text { Grupo Televisa, } \\
\text { S.A.B. CPOs }\end{array}$ & (TLEVISACPO.MX) \\
\hline $\begin{array}{l}\text { Industrias Peñoles, } \\
\text { S.A.B. de C.V. }\end{array}$ & (PE\&OLES.MX) & $\begin{array}{l}\text { Kimberly-Clark de } \\
\text { México, S. A. B. de C. V. }\end{array}$ & (KIMBERA.MX) \\
\hline $\begin{array}{l}\text { Mexichem, } \\
\text { S.A.B. de C.V. }\end{array}$ & (MEXCHEM.MX) & & \\
\hline
\end{tabular}

\subsection{Modelo de regresión lineal de datos panel}

Un panel se define como una muestra representativa de la población que permanece constante en el tiempo y que se estudia de forma repetitiva y periódica a intervalos regulares de tiempo, Yustas López (2014). De acuerdo con Baltagi (2008), dentro de un conjunto de datos panel, se tienen observaciones de series temporales sobre una muestra de unidades individuales. Para una variable $Y_{i t}$, se supone que se tiene $i=1, \ldots N$, observaciones de corte transversal y $t=1, \ldots T$ observaciones temporales, donde $i$ y $t$ hacen referencia a los individuos y al periodo de tiempo, respectivamente. La forma habitual en la que se presentan los modelos de datos de panel es:

$$
Y_{i t}=\alpha_{i t}+X_{i t} \beta+u_{i t} \quad i=1, \ldots N, \quad t=1, \ldots T
$$

El subíndice i denota al individuo y el subíndice t denota el periodo de tiempo al que está referida la observación del individuo, $\alpha$ es un vector de interceptos de $\mathrm{n}$ parámetros, $\beta$ es un vector de $\mathrm{k}$ parámetros y $X_{i t}$ la i-ésima observación al momento $t$ para las $k$ variables explicativas, $x$ representa entonces la variable independiente y el termino u al error estimado. El parámetro a estimar $\beta$ no está acompañado de ningún 
subíndice, porque se considera que permanece constante en el tiempo y que es el mismo para todos los individuos. Para el caso de regresión lineal múltiple con datos panel, la ecuación queda de la forma:

$$
Y_{i t}=\alpha_{i t}+X_{K i t} \beta_{k}+u_{i t}
$$

donde $Y_{i t}$ es el vector que contiene la información del individuo $i$ en todo $t$.

\subsection{Especificación del modelo}

Se analizan los datos financieros de las 13 empresas para determinar su impacto en los componentes trimestrales del IPC Sustentable de 2015 a 2018, publicados por la BMV (DSC y sus tres dimensiones IA, IS e IGC). Se tienen 15 trimestres que generaron 195 observaciones en un modelo de panel de datos con Mínimos Cuadrados Ordinarios Agrupados, utilizando para el análisis el paquete estadístico EVIEWS 9.

A continuación, se muestran las ecuaciones que conforman el modelo de regresión en el que se analiza si los indicadores financieros retorno sobre los activos (ROA), Quick ratio (quickr), tamaño total de los activos (tactivos), margen de beneficios antes de intereses, impuestos, depreciación y amortización (ebitdam), y ratio del puntaje de la z-score (zaltman), de las empresas que forman el IPC sustentable de la BMV impactan al índice ambiental (IA), índice de responsabilidad social (IS), índice de gobierno corporativo (IGC) y en general al Desempeño Sustentable Corporativo (DSC).

Modelo de regresión lineal para el desempeño sustentable corporativo:

$$
D S C=\alpha+\beta_{2} R O A+\beta_{3} \text { ebitdam }+\beta_{4} q u i c k r+\beta_{5} \text { tactivos }+\beta_{6} \text { Zaltman }
$$

Ecuación de regresión lineal para el desempeño ambiental:

$$
I A=\alpha+\beta_{2} R O A+\beta_{3} \text { ebitdam }+\beta_{4} \text { quickr }+\beta_{5} \text { tactivos }+\beta_{6} \text { Zaltman }
$$

Ecuación de regresión lineal para la responsabilidad social:

$$
I S=\alpha+\beta_{2} R O A+\beta_{3} \text { ebitdam }+\beta_{4} \text { quickr }+\beta_{5} \text { tactivos }+\beta_{6} \text { Zaltman }
$$

Ecuación de regresión lineal para el gobierno corporativo:

$$
I G C=\alpha+\beta_{2} R O A+\beta_{3} \text { ebitdam }+\beta_{4} \text { quickr }+\beta_{5} \text { tactivos }+\beta_{6} \text { Zaltman }
$$

Las variables financieras consideradas en los modelos son las que indican crecimiento y estabilidad de la empresa, y presentan menor correlación. Debido a la variación de tamaño entre las empresas (medido por sus activos) y las variaciones que puedan existir en los trimestres del estudio, se aplicaron efectos fijos en las secciones cruzadas y en los periodos, para la determinación de los resultados.

\section{Análisis de Resultados}

Al aplicar los modelos de panel con Mínimos Cuadrados Ordinarios Agrupados y efectos fijos en secciones cruzadas y periodos, al Desempeño Sustentable Corporativo (DSC) y cada uno de sus subíndices: índice ambiental (IA), índice de responsabilidad social (IS) e índice de gobierno corporativo (IGC); de la muestra de las empresas que cotizan en el IPC Sustentable de la BMV; se obtiene que los modelos tienen un buen ajuste, aunque algunas de las variables no son significativas. Como se muestra en la tabla 4. 
Tabla 4. Resultados de los modelos de panel para el desempeño sustentable de las empresas que cotizan en

\begin{tabular}{|c|c|c|c|c|c|c|c|c|}
\hline & \multicolumn{2}{|c|}{ DSC IPC Sustentable de la BMV } & \multicolumn{2}{c|}{ IS } & \multicolumn{2}{c|}{ IA } & \multicolumn{2}{c|}{ IGC } \\
& Coefficient & Prob. & Coefficient & Prob. & Coefficient & Prob. & Coefficient & Prob. \\
\hline EBITDAM & 5.123320 & 0.438900 & -4.126045 & 0.686000 & 18.682040 & 0.071800 & 0.813962 & 0.937000 \\
\hline ROA & 0.583809 & 0.968900 & 20.831790 & 0.367100 & -14.446670 & 0.249400 & -4.633689 & 0.063200 \\
\hline TACTIVOS & -0.000024 & 0.000600 & -0.000024 & 0.021800 & -0.000025 & 0.536300 & -0.000022 & 0.842400 \\
\hline ZALTMAN & -2.517397 & 0.000100 & -1.902629 & 0.056800 & -3.635428 & 0.018000 & -2.014133 & 0.041400 \\
\hline QUICKR & 0.576181 & 0.391300 & 0.983947 & 0.342800 & -1.209659 & 0.000400 & 1.954256 & 0.046000 \\
\hline C & 75.143510 & 0.000000 & 71.042330 & 0.000000 & 74.752060 & 0.000000 & 79.636150 & 0.000000 \\
\hline R-cuadrada & \multicolumn{2}{|c|}{0.931327} & 0.904289 & 0.873683 & 0.901950 \\
\hline Akaike info criterion & 4.868651 & \multicolumn{2}{|c|}{5.735890} & \multicolumn{2}{|c|}{5.759615} & & 5.755855 \\
\hline Durbin-Watson stat & 0.631319 & \multicolumn{2}{|c|}{0.474673} & & 0.606004 & & 0.424904 \\
\hline
\end{tabular}

Fuente: elaboración propia con base en EVIEWS 9

Los resultados muestran que ROA es una variable positiva para Desempeño Sustentable Corporativo (DSC) y para el índice ambiental (IA), negativa para el índice de responsabilidad social (IS) y para el Desempeño Sustentable Corporativo (DSC); sin embargo, en todos los modelos es no significativa, en línea con lo obtenido en el análisis de estudios previos: Hindley \& Buys (2012), Ameer \& Othman (2012), Bayoud \& Kavanagh (2012), Eccles, Krzus, Rogers, \& Serafeim (2012), Burhan \& Rahmanti (2012), Venanzi (2013) y Motwani \& Pandya (2016). Las variables quickr, tactivos, ebitdam y zaltman no fueron analizadas en estudios previos.

La variable quickr fue negativa y significativa para IS, para los otros modelos fue positiva y sólo ligeramente significativa para IGC; mientras que ebitdam es negativa sólo para IA y ligeramente significativa sólo para IS. No se tomarán en cuenta en el modelo final por estandarización de las variables significativas en los cuatro modelos.

Las variables que resultan significativas en los cuatro modelos son tactivos y zaltman, tienen relación negativa para los cuatro índices; el intercepto es significativo y presenta relación positiva en los modelos. Los más representativos son los que se muestran en la Tabla 5.

Tabla 5. Resultados de los modelos de regresión lineal para el desempeño sustentable de las empresas que

\begin{tabular}{|c|c|c|c|c|c|c|c|c|}
\multicolumn{1}{|c}{ cotizan en el IPC Sustentable de la BMV } \\
& \multicolumn{2}{|c|}{ DSC } & \multicolumn{2}{c|}{ IA } & \multicolumn{2}{c|}{ IS } & \multicolumn{2}{c|}{ IGC } \\
& Coefficient & Prob. & Coefficient & Prob. & Coefficient & Prob. & Coefficient & Prob. \\
\hline TACTIVOS & -0.00002 & 0.00080 & -0.00002 & 0.02370 & -0.00003 & 0.01000 & -0.00002 & 0.10210 \\
\hline ZALTMAN & -2.04953 & 0.00020 & -1.27422 & 0.12340 & -2.93217 & 0.00070 & -1.94221 & 0.02120 \\
\hline C & 75.24380 & 0.00000 & 70.40517 & 0.00000 & 74.92639 & 0.00000 & 80.39983 & 0.00000 \\
\hline R-squared & \multicolumn{2}{|c|}{0.93022} & \multicolumn{2}{|c|}{0.90309} & 0.86830 & 0.89981 \\
\hline Akaike info criterion & \multicolumn{2}{|c|}{4.85387} & \multicolumn{2}{|c|}{5.7176} & 5.770614 & 5.746727 \\
\hline Durbin-Watson stat & \multicolumn{2}{|c|}{0.63050} & \multicolumn{2}{|c|}{0.46966} & 0.58422 & 0.40085 \\
\hline
\end{tabular}

Fuente: elaboración propia con base en EVIEWS 9

Los resultados muestran un buen ajuste en todos los modelos y debido a las distintas características de tamaño y actividad de las empresas que forman la muestra no existe igualdad de medias y varianzas en los cortes transversales del modelo; por lo que considerar efectos fijos resulta adecuado. Podemos observar que las variables son significativas, principalmente en el modelo de Desempeño Sustentable Corporativo (DSC) y para el Índice de Responsabilidad Social (IS); así mismo, se puede observar que existe evidencia de la auto correlación positiva en los modelos, como se observa también con el Gráfico 1. 


\begin{tabular}{|c|c|c|c|c|c|c|c|}
\hline \multicolumn{8}{|c|}{ Correlogram of DSC } \\
\hline \multicolumn{8}{|c|}{$\begin{array}{l}\text { Date: 06/10/19 Time: } 15: 49 \\
\text { Sample: } 201501201803 \\
\text { Included observations: } 195\end{array}$} \\
\hline Autocorrelation & Partial C & orrelation & & $A C$ & PAC & Q-Stat & Prob \\
\hline $1 \longmapsto$ & 1 & $\square$ & 1 & 0.910 & 0.910 & 164.12 & 0.000 \\
\hline 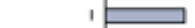 & 1 & 1 & 2 & 0.829 & -0.002 & 300.77 & 0.000 \\
\hline $1 \longmapsto$ & & 1 & 3 & 0.749 & -0.030 & 413.02 & 0.000 \\
\hline $1 \longmapsto$ & & 1 & 4 & 0.670 & -0.041 & 503.32 & 0.000 \\
\hline $1 \rightleftharpoons$ & 1 & 11 & 5 & 0.603 & 0.022 & 576.77 & 0.000 \\
\hline $1 \longmapsto$ & & 1 & 6 & 0.537 & -0.027 & 635.44 & 0.000 \\
\hline $1 巴$ & If & 1 & 7 & 0.472 & -0.039 & 680.96 & 0.000 \\
\hline $1 \longmapsto$ & 10 & 1 & 8 & 0.404 & -0.062 & 714.44 & 0.000 \\
\hline $1 \sqsubseteq$ & it & 1 & 9 & 0.339 & -0.025 & 738.15 & 0.000 \\
\hline 1曰 & 16 & 1 & 10 & 0.273 & -0.053 & 753.62 & 0.000 \\
\hline 口 & 1 & 1 & 11 & 0.212 & -0.022 & 763.00 & 0.000 \\
\hline 吕 & 14 & 1 & 12 & 0.150 & -0.056 & 767.75 & 0.000 \\
\hline
\end{tabular}

\begin{tabular}{|c|c|c|c|c|c|c|c|}
\hline \multicolumn{8}{|c|}{ Correlogram of IS } \\
\hline \multicolumn{8}{|c|}{$\begin{array}{l}\text { Date: 06/10/19 Time: } 15: 51 \\
\text { Sample: } 201501201803 \\
\text { Included observations: } 195\end{array}$} \\
\hline Autocorrelation & Partial Co & orrelation & & $A C$ & PAC & Q-Stat & Prob \\
\hline $1 巨$ & , & $\square$ & 1 & 0.904 & 0.904 & 161.98 & 0.000 \\
\hline 1 & & 1 & 2 & 0.814 & -0.020 & 293.99 & 0.000 \\
\hline ए & 1 & 1 & 3 & 0.733 & -0.000 & 401.52 & 0.000 \\
\hline $1 \rightleftharpoons$ & & 1 & 4 & 0.653 & -0.036 & 487.39 & 0.000 \\
\hline 啊 & & 1 & 5 & 0.578 & -0.021 & 554.98 & 0.000 \\
\hline 巴 & & 1 & 6 & 0.501 & -0.055 & 606.08 & 0.000 \\
\hline $1 \Xi$ & & 1 & 7 & 0.429 & -0.023 & 643.75 & 0.000 \\
\hline $1 \Xi$ & & 1 & 8 & 0.356 & -0.056 & 669.79 & 0.000 \\
\hline $1 \square$ & 1 & 1 & 9 & 0.290 & -0.014 & 687.11 & 0.000 \\
\hline $1 曰$ & id & 1 & 10 & 0.218 & -0.077 & 697.02 & 0.000 \\
\hline 㢄 & 1 & 1 & 11 & 0.157 & -0.002 & 702.16 & 0.000 \\
\hline$\sqrt{2}$ & 1 & $b_{1}$ & 12 & 0.110 & 0.028 & 704.70 & 0.000 \\
\hline
\end{tabular}

\begin{tabular}{|c|c|c|c|c|c|c|c|}
\hline \multicolumn{8}{|c|}{ Correlogram of IA } \\
\hline \multicolumn{8}{|c|}{$\begin{array}{l}\text { Date: 06/10/19 Time: } 15 \cdot 54 \\
\text { Sample: } 2015012018 Q 3 \\
\text { Included observations: } 195\end{array}$} \\
\hline Autocorrelation & Partial Co & orrelation & & $A C$ & PAC & Q-Stat & Prob \\
\hline $1 E$ & it & $\square$ & 1 & 0.909 & 0.909 & 163.48 & 0.000 \\
\hline ש & & 1 & 2 & 0.819 & -0.036 & 297.07 & 0.000 \\
\hline $1 \rightleftharpoons$ & & 1 & 3 & 0.732 & -0.037 & 404.28 & 0.000 \\
\hline : & & 11 & 4 & 0.651 & -0.012 & 489.63 & 0.000 \\
\hline 䟚 & 1 & 1 & 5 & 0.580 & 0.006 & 557.68 & 0.000 \\
\hline ( & 1 & 1 & 6 & 0.518 & 0.009 & 612.23 & 0.000 \\
\hline ש & & d 1 & 7 & 0.456 & -0.040 & 654.68 & 0.000 \\
\hline ש & & 1 & 8 & 0.382 & -0.109 & 684.59 & 0.000 \\
\hline$\square$ & 17 & 1 & 9 & 0.314 & -0.009 & 704.99 & 0.000 \\
\hline 呵 & & d & 10 & 0.249 & -0.033 & 717.89 & 0.000 \\
\hline 口 & & $d$ & 11 & 0.184 & -0.057 & 724.92 & 0.000 \\
\hline i & 10 & d & 12 & 0.124 & -0.025 & 728.13 & 0.000 \\
\hline
\end{tabular}

\begin{tabular}{|c|c|c|c|c|c|c|c|}
\hline \multicolumn{8}{|c|}{ Correlogram of IGC } \\
\hline \multicolumn{8}{|c|}{$\begin{array}{l}\text { Date: 06/10/19 Time: } 15: 55 \\
\text { Sample: } 201501201803 \\
\text { Included observations: } 195\end{array}$} \\
\hline Autocorrelation & Partial Co & orrelation & & $A C$ & PAC & Q-Stat & Prob \\
\hline $1 \longmapsto$ & if & $\square$ & 1 & 0.915 & 0.915 & 165.76 & 0.000 \\
\hline 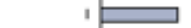 & & 1 & 2 & 0.819 & -0.110 & 299.33 & 0.000 \\
\hline$\square$ & & 1 & 3 & 0.725 & -0.038 & 404.53 & 0.000 \\
\hline P & 1 & 1 & 4 & 0.638 & -0.014 & 486.37 & 0.000 \\
\hline $1 \rightleftharpoons$ & & וg - l & 5 & 0.571 & 0.069 & 552.29 & 0.000 \\
\hline ए & & 1 & 6 & 0.507 & -0.039 & 604.58 & 0.000 \\
\hline $1 巴$ & & 1 & 7 & 0.447 & -0.021 & 645.39 & 0.000 \\
\hline (巴 & i) & 1 & 8 & 0.392 & -0.007 & 676.90 & 0.000 \\
\hline E & & 1 & 9 & 0.332 & -0.062 & 699.62 & 0.000 \\
\hline 㕷 & id & 1 & 10 & 0.267 & -0.071 & 714.39 & 0.000 \\
\hline 㕷 & 11 & 1 & 11 & 0.205 & -0.020 & 723.18 & 0.000 \\
\hline 吅 & if & 1 & 12 & 0.146 & -0.030 & 727.68 & 0.000 \\
\hline
\end{tabular}

Gráfico 1. Análisis de auto correlación de los modelos DSC e IS

Fuente: elaboración propia con base en EVIEWS 9

Un resultado interesante es que en todos los modelos las variables explicativas tactivos y zaltman, tienen un efecto inverso en los índices de sustentabilidad. Lo que indica que entre mayores son sus activos o su nivel de solvencia menos impactan a sus índices sustentables; lo anterior sugiere que a las empresas que cotizan en el IPC Sustentable de la BMV les representa un gasto adicional cumplir con los índices de sustentabilidad.

En el análisis de los residuos de los modelos finales, las pruebas de igualdad de varianza no muestran heteroscedasticidad entre secciones cruzadas y las gráficas Q-Q se muestran en el Gráfico 2.
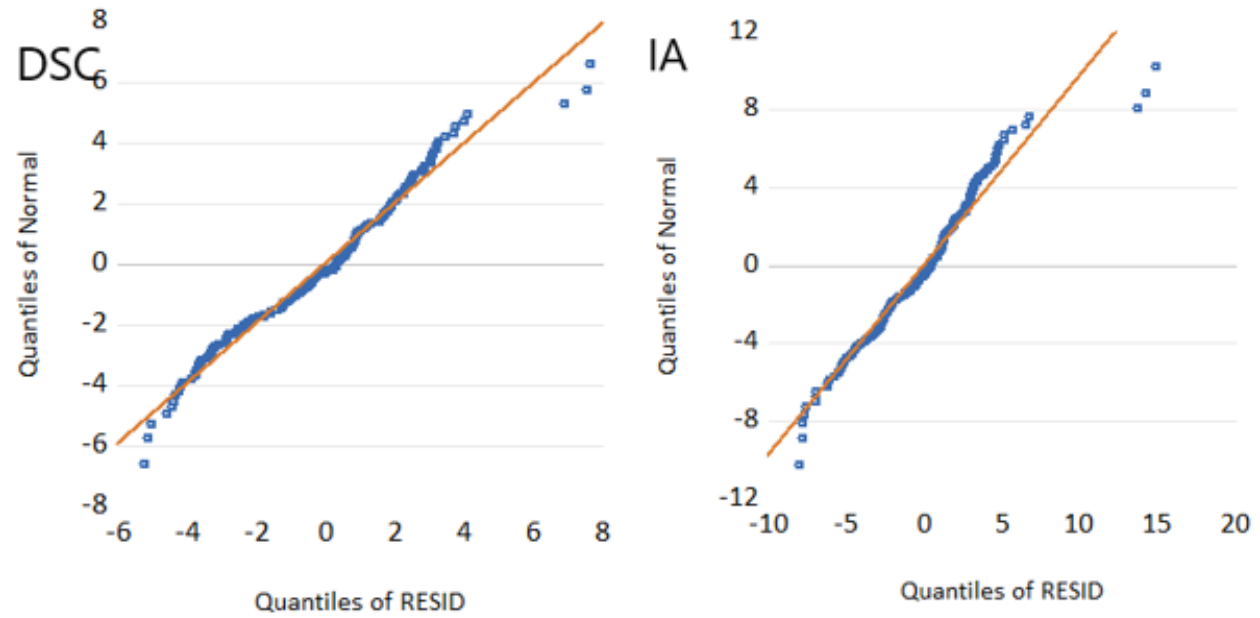

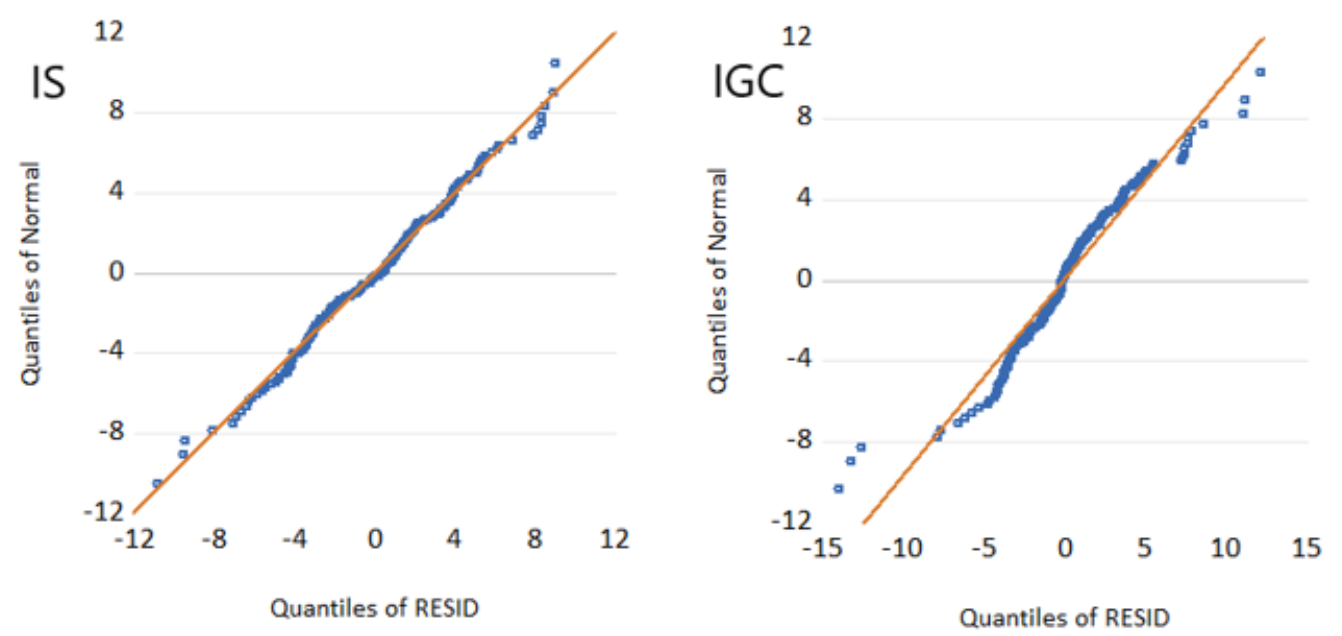

Gráfico 2. Gráfica Q-Q del modelo DSC

Fuente: elaboración propia con base en EVIEWS 9

Por lo que podemos concluir que el impacto de los indicadores financieros es negativo a los índices de sustentabilidad de las empresas que cotizan en el IPC Sustentable de la BMV.

\section{Conclusiones y consideraciones finales}

El objetivo de la presente investigación, fue determinar si los indicadores financieros: retorno sobre los activos (ROA), Quick ratio (quickr), tamaño total de los activos (tactivos), margen de beneficios antes de intereses, impuestos, depreciación y amortización (ebitdam), y ratio del puntaje de la z-score (zaltman), de las empresas que forman el IPC sustentable de la BMV impactan al índice ambiental (IA), índice de responsabilidad social (IS), índice de gobierno corporativo (IGC) y en general al Desempeño Sustentable Corporativo (DSC), por medio de un modelo de panel con Mínimos Cuadrados Ordinarios Agrupados y efectos fijos en secciones cruzadas y periodos. Se analizaron los datos trimestrales de una muestra de 13 empresas en el periodo 2015-2018, lo que generó 15 trimestres y 195 observaciones; para el análisis se utilizó el paquete estadístico EVIEWS 9.

Podemos concluir que ROA es una variable positiva para Desempeño Sustentable Corporativo (DSC) y para el índice ambiental (IA), negativa para el índice de responsabilidad social (IS) y para el Desempeño Sustentable Corporativo (DSC); sin embargo, en todos los modelos es no significativa, en línea con lo obtenido en el análisis de estudios. Además, se presenta buen ajuste en los modelos y debido a las distintas características de tamaño y actividad de las empresas que forman la muestra no existe igualdad de medias y varianzas en los cortes transversales del modelo; por lo que considerar efectos fijos resulta adecuado y existe evidencia de la auto correlación positiva.

Las variables más significativas del estudio fueron tactivos y zaltman; los resultados determinan que existe una relación inversa entre estas variables y las variables dependientes DSC, IA, IS e IGC. Lo que implica que, las empresas que cotizan en el IPC Sustentable de la BMV tienen un costo extra por formar parte de dicho índice y que no se acepta la hipótesis de que a mayor desempeño financiero mejores niveles de sustentabilidad.

Las principales limitaciones de la investigación son, el bajo número de observaciones, por ser un índice relativamente nuevo y que sólo algunas empresas tenían datos públicos y fueron consistentes con su evaluación de sustentabilidad en los trimestres de análisis. Es recomendable que, transcurridos unos años, analizar el total de las empresas con más trimestres de historia, para comprobar que los resultados son consistentes para 
todas las empresas que cotizan en el índice; así como la aplicación de otro tipo de modelos para verificar si es posible mejorar los resultados.

Otro punto pendiente de analizar, es si existen posibles ventajas fiscales, de mercado u otras que tengan un impacto directo con la sustentabilidad de las empresas que cotizan en el IPC sustentable de la BMV.

\section{Referencias}

[1] Abrams, F. (1951). Management's responsibilities in a complex world. Harvard Business Review, 29(3), 29-34.

[2] Aggarwal, P. (2013). Impact of Sustainability Performance of Company on its Financial Performance: A Study of Listed Indian Companies. Global Journal of Management and Business Research (C: Finance) Volume 13, Issue 11, Version 1.0, 13(11), Available at SSRN: https://ssrn.com/abstract=3131923.

[3] Ameer, R., \& Othman, R. (2012). Sustainability Practices and Corporate Financial Performance: A Study Based on the Top Global Corporations. Journal of Business Ethics, 108, 61-79. DOI: 10.1007/s10551-011-1063-y.

[4] Artiach, T., Lee, D., Nelson, D., \& Walker, J. (2010). The determinants of corporate sustainability performance. Accounting \& Finance, 50(1), 31-51. https://doi.org/10.1111/j.1467-629X.2009.00315.x. doi:10.1111/j.1467629X.2009.00315.x

[5] Baltagi, B. H. (2008). Econometric analysis of panel data. Chichester, West Sussex: John Wiley \& Sons.

[6] Bansal, P. (2005). Evolving sustainably: A longitudinal study of corporate sustainable development. Strategic Management Journal, 26(3), 197-218. Retrieved from https://www.jstor.org/stable/20142218

[7] Barcellos de Paula, L. (2010). Modelos de gestión aplicados a la sostenibilidad empresarial. Barcelona, España: Universitat de Barcelona. Retrieved from http://diposit.ub.edu/dspace/bitstream/2445/35386/2/LBP_ TESIS.pdf

[8] Bayoud, N. S., \& Kavanagh, M. (2012). Corporate Social Responsibility Disclosure: Evidence from Libyan Managers. Global Journal of Business Research, v. 6 (5), 73-83, Available at SSRN: https://ssrn.com/abstract $=2146106$.

[9] Bergman, M., Bergman, Z., \& Berger, L. (2017). An Empirical Exploration, Typology, and Definition of Corporate Sustainability. Sustainability, 9(5), 753. doi:10.3390/su9050753

[10] Bhaduri, S., \& Selarka, E. (2016). Corporate governance and corporate social responsibility of Indian companies. Singapore: Springer .

[11] Bolsa Mexicana de Valores . (2014). Nota Metodológica del IPC Sustentable . México, D.F.: Bolsa Mexicana de Valores.

[12] BOVESPA. (2019, 05 02). ISEB3. Retrieved from What is ISE B3?: http://iseb3.com.br/o-que-e-o-ise

[13] Bowen, H. (1953). Social Responsibilities of the Businessman. New York: Harper \& Brothers.

[14] Brammer, S., Brooks, C., \& Pavelin, S. (2006). Corporate Social Performance and Stock Returns: UK Evidence from Disaggregate Measures. Financial Management, 97-116. https://doi.org/10.1111/j.1755053X.2006.tb00149.x.

[15] Burhan, A. H., \& Rahmanti, W. (2012). THE IMPACT OF SUSTAINABILITY REPORTING ON COMPANY PERFORMANCE. Journal of Economics, Business, and Accountancy Ventura, 15(2), 257-272, DOI: http://dx.doi.org/10.14414/jebav.v15i2.79.

[16] BYMA. (2019, 05 07). Noticias. Retrieved from Índice de Sustentabilidad del mercado argentino: https://www.byma.com.ar/noticias/byma-lanza-el-primer-indice-de-sustentabilidad-del-mercado-argentino/

[17] Calvente, A. M. (2007, Junio). El concepto moderno de sustentabilidad. UAIS Sustentabilidad., 1(1), 1-7. Retrieved from http://www.sustentabilidad.uai.edu.ar/pdf/sde/UAIS-SDS-100-002 \%20- \%20Sustentabilidad.pdf

[18] Carneiro, M. (2004). La responsabilidad social corporativa interna. Madrid: Esic editorial. 
[19] Carroll, A., \& Shabana, K. (2010). The Business Case for Corporate Social Responsibility: A Review of Concepts, Research and Practice. International Journal of Management Reviews, 12(1), 85-105. doi:10.1111/j.14682370.2009.00275.x

[20] Chouinard, Y., Ellison, J., \& Ridgeway, R. (2011). The Sustainable Economy Harvard Business Review. Harvard Business Review, 89(10), 52-62.

[21] CLP, G. (2019, 05 07). Sustainability Ratings>. Retrieved from Dow Jones Sustainability Asia Pacific Index: https://www.clpgroup.com/en/sustainability/sustainability-ratings/dow-jones-sustainability-asia-pacific-index

[22] D'Amato, A., Henderson, S., \& Florence, S. (2009). Corporate social responsibility and sustainable business. A Guide to Leadership Tasks and Functions. Center for Creative Leadership: Greensboro, North Carolina. Retrieved from http://www.retawprojects.com/uploads/CorporateSocialResponsibility.pdf

[23] Dahlsrud, A. (2008). How corporate social responsibility is defined: an analysis of 37 definitions. Corporate social responsibility and environmental management, 15(1), 1-13. doi: https://doi.org/10.1002/csr.132

[24] De la Torre, O., \& Martínez, M. (2015). Revisión de la inversión sustentable en la bolsa mexicana durante periodos de crisis. Revista mexicana de economía y finanzas, 10(2), 115-130.

[25] Delgadillo, J. L. (2007). EL DESARROLlO SUSTENTABLE EN MÉXICO (1980-2007). (C. d. DGSCA-UNAM, Ed.) Revista Digital Universitaria, 9(3), 1-13. Retrieved from http://www.revista.unam.mx/vol.9/num3/art14/art14.pdf

[26] Deloitte. (2012). Panorama global de la sustentabilidad y su impacto en México. Galaz, Yamazaki, Ruiz Urquiza, S.C. Retrieved from https://www2.deloitte.com/content/dam/Deloitte/mx/Documents/risk/ Sustentabilidad/mx (es-mx)POV_Sustentabilidad_panorama.pdf

[27] Deloitte. (2012). Panorama global de la sustentabilidad y su impacto en México. Retrieved from earthgonomic: http://www.earthgonomic.com/biblioteca/2012_DELOITTE_Sustentabilidad_panorama_mx.pdf

[28] Dhaliwal, D. S., Zhen Li, O., Tsang, A., \& Yang, Y. G. (2011). Voluntary nonfinancial disclosure and the cost of equity capital: The initiation of corporate social responsibility reporting. The Accounting Review 86 (1), 59-100, https://doi.org/10.2308/accr.00000005.

[29] Docekalova, M., Kocmanová, A., \& Koleňák, J. (2015). Determination of Economic Indicators in the Context of Corporate Sustainability Performance. Business: Theory and Practice. 16(1), 16(1), 15-24. DOI: 10.3846/btp.2015.450. doi:doi:10.3846/btp.2015.450

[30] Eccles, R., Krzus, M. P., Rogers, J., \& Serafeim, G. (2012). The Need for Sector-Specific Materiality and Sustainability Reporting Standards. Journal of Applied Corporate Finance 24(2), DOI: 10.1111/j.1745-6622.2012.00380.x.

[31] ETHIBEL, F. (2019, 05 07). Who are we? Retrieved from History: http://www.forumethibel.org/content/geschiedenis.html

[32] EY. (2019, 05 07). Sustainability Alert. Retrieved from 13 empresas mexicanas forman parte del Dow Jones Sustainability MILA Pacific: https://www.ey.com/Publication/vwLUAssets/ey-13-empresas-mexicanas-formanparte-del-dow-jones-sustainability-mla-pacific-alliance-index-2018/\$FILE/ey-13-empresas-mexicanas-formanparte-del-dow-jones-sustainability-mla-pacific-alliance-index-2018.pdf

[33] Ferruz Agudo, L., Marco Sanjuán, I., \& Muñoz Sánchez, F. (2009). Fondos de inversión éticos, ecológicos y socialmente responsables en Europa (1999-2007). Aposta, Revista de Ciencias Sociales(48), 1-28.

[34] Friedman, M. (1970). A Theoretical Framework for Monetary Analysis. Journal of Political Economy, 78(2), 193-238. Retrieved from https://www.jstor.org/stable/1830684

[35] FTSERussell. (2019, 05 07). FTSE4Good. Retrieved from FTSE4Good Index Series: https://www.ftse.com/products/indices/FTSE4Good

[36] Gladwin, T., Kennelly, J., \& Krause, T.-S. (1995). Shifting Paradigms for Sustainable Development: Implications for Management Theory and Research. The Academy of Management Review, 20(4), 874-907. doi:10.2307/258959

[37] Goodpaster, K., Rodbourne, D., Hernke, B., \& Betzler, L. (2010). Corporate Social Responsibility-The shape of a History, 1945-2004. Minneapolis: Center for Ethical Business Cultures. CEBC. 
[38] Haffar, M., \& Searcy, C. (2017). Classification of Trade-offs Encountered in the Practice of Corporate Sustainability. Journal of Business Ethics, 140(3), 495-522. https://doi.org/10.1007/s10551-015-2678-1.

[39] Hamidu, A., Haron, M., \& Amran, A. (2015). Corporate Social Responsibility: A Review on definitions, core characteristics and theoretical perspectives. Retrieved from Munich Personal RePEc Archive: https://mpra.ub.unimuenchen.de/75040/

[40] Hindley, T., \& Buys, P. (2012). Integrated Reporting Compliance With The Global Reporting Initiative Framework: An Analysis Of The South African Mining Industry. International Business \& Economics Research Journal, 11(11), 1249-1260, DOI: 10.19030/iber.v11i11.7372.

[41] INC, M. (2019, 05 02). MSCI ACWI. Retrieved from MSCI ACWI ESG LEADERS INDEX (USD): https://www.msci.com/documents/10199/2258707f-8f08-4722-b68e-564be3a064f1

[42] Indices, S. D. (2019, 05 07). Índices. Retrieved from S\&P/BMV IPC Sustentable: https://espanol.spindices.com/indices/equity/sp-bmv-ipc-sustainable

[43] Jones, S. (2005). Notes of The University of Sydney Pacioli Society. ABACUS, 41(2), 211-216, https://onlinelibrary.wiley.com/doi/epdf/10.1111/j.1467-6281.2005.00176.x.

[44] Kocmanova, A., Simanaviciene, Z., \& Docekalova, M. (2015). Predictive model for measuring sustainability of manufacturing companies. Engineering Economics. 26(4), 26(4), 442-451. https://doi.org/10.5755/j01.ee.26.4.11480. doi:http://dx.doi.org/10.5755/j01.ee.26.4.11480

[45] Leal, F., González, R., \& Romo , L. (2014). Sustentabilidad de las Pymes en base al Índice de Precios y Cotizaciones (IPC Sustentable) En México y el Financiamiento Verde. Aguascalientes : UNIVERSIDAD AUTONOMA DE AGUASCALIENTES .

[46] Linnenluecke, M., \& Griffiths, A. (2010). Corporate sustainability and organizational culture. Journal of World Business, 45(4), 357-366.

[47] Lockett, A., Moon , J., \& Visser, W. (2006). Corporate Social Responsibility in Management Research: Focus, Nature, Salience and Sources of Influence. Journal of Management Studies, 43(1), 115-136.

[48] Lourenço, I., \& Branco, M. (2013). Determinants of Corporate sustainability performance in emerging markets: the Brazilian case. Journal of Cleaner Production. Vol. 57, 57, $134-141$. https://doi.org/10.1016/j.jclepro.2013.06.013. doi:https://doi.org/10.1016/j.jclepro.2013.06.013

[49] Lourenço, I., Branco, M., Curto, J., \& Eugénio, T. (2012). How Does the Market Value Corporate Sustainability Performance? Journal of Business Ethics, 108(4), 417-428, DOI:10.1007/s10551-011-1102-8.

[50] Low, M. (2016). Corporate social responsibility and the evolution of internal corporate social responsibility in 21st century. Asian Journal of Social Sciences and Management Studies, 3(1), 56-74.

[51] Mackenzie, C., Rees, W., \& Rodionova, T. (2013). Do Responsible Investment Indices Improve Corporate Social Responsibility? FTSE4Good's Impact on Environmental Management. Corporate Governance: An International Review, 21(5), 495-512. doi:https://doi.org/10.1111/corg.12039

[52] Marichalar de Corral, B. (2014). Índices bursátiles sostenibles y otros criterios para "medir"la sostenibilidad en la empresa. Retrieved from Universidad Pontificia Comillas: https://repositorio.comillas.edu/rest/bitstreams/68/retrieve

[53] Moneva, J. M., Archel, P., \& Correa, C. (2006). GRI and the camouflaging of corporate unsustainability. Accounting Forum, 30:2, 121-137, DOI: 10.1016/j.accfor.2006.02.001.

[54] Montiel , I., \& Delgado-Ceballos, J. (2014). Defining and Measuring Corporate Sustainability: Are We There Yet? Organization \& Environment, 27(2), 113-139. doi:10.1177/1086026614526413

[55] Motwani, S. S., \& Pandya, H. B. (2016). Evaluating the Impact of Sustainability Reporting on Financial Performance of Selected Indian Companies. International Journal of Research in IT and Management. Volume 6, Issue $2,6(2), 14-23$.

[56] MSCI. (2019, 05 02). ENVIRONMENTAL, SOCIAL, AND GOVERNANCE (ESG). Retrieved from MSCI KLD 400 SOCIAL INDEX: https://www.msci.com/msci-kld-400-social-index 
[57] Nigro, C., Iannuzzi, E., Cortese, F., \& Petracca, M. (2015). The relationship between corporate social responsibility and financial performance. An empirical analysis on a sample of italian listed firms. 8th Annual Conference of the EuroMed Academy of Business (pp. 1456-1469). Verona, Italy: EuroMed Press. DOI: 10.13140/RG.2.1.2085.9364. doi:10.13140/RG.2.1.2085.9364

[58] Pinillos, A., \& Fernández , J. (2011). De la RSC a la sostenibilidad corporativa: una evolución necesaria para la creación de valor. Harvard Deusto business review, 207, 4-21.

[59] Ramírez, A., Sanchéz, J. M., \& García, A. (2004). El Desarrollo Sustentable: Interpretación y Análisis. Revista del Centro de Investigación. Universidad La Salle, 6(21), 55-59. Retrieved from http://www.redalyc.org/pdf/342/34202107.pdf

[60] Renneboog, L., Horst, J., \& Zhang, C. (2007). Socially Responsible Investments: Methodology, Risk Exposure and Performance. Retrieved from European Corporate Governance Institute: http://ssrn.com/abstract_id=985267

[61] Reverte, C. J. (2009). Determinants of Corporate Social Responsibility Disclosure Ratings by Spanish Listed Firms. Journal of Business Ethics, 88(2), 88: 351. https://doi.org/10.1007/s10551-008-9968-9. doi:10.1007/s10551-008-9968-9

[62] ROBECOSAM. (2019, 05 02). Dow Jones Sustainability Indices. Retrieved from DJSI index family: https://www.sustainability-indices.com/index-family-overview/djsi-index-family.html

[63] Russell, F. (2019, 09 04). All Indexes. Retrieved from FTSE4Good Index Series: https://www.ftserussell.com/products/indices/FTSE4Good

[64] S\&P. (2019, 05 02). S\&P Dow Jones Indices. Retrieved from Dow Jones Sustainability Chile Index (CLP): https://espanol.spindices.com/indices/equity/dow-jones-sustainability-chile-index-clp

[65] S\&P Dow Jones Indices. (2018, Noviembre 21). S\&P/BMV Indices Metodología. Retrieved from https://espanol.spindices.com/indices/equity/sp-bmv-ipc-sustainable

[66] S\&P, D. J. (2019, 05 07). Indices by Region. Retrieved from Pacific Alliance: https://us.spindices.com/regionalexposure/americas/pacific-alliance

[67] Saavedra, M. (2011). La responsabilidad social empresarial y las finanzas. Cuadernos de Administración, Universidad de Valle, 27(46).

[68] Schueth, S. (2003). Socially responsible investing in the United States. Journal of business ethics,, 43(3), $189-194$. Retrieved from https://link.springer.com/content/pdf/10.1023/A:1022981828869.pdf

[69] Soytas, M., Denizel, M., \& Usar, D. (2017). Corporate Sustainability: Empirical Evidence of Causality on Financial Performance. Working Paper, DOI: 10.13140/RG.2.2.29245.15846. doi:10.13140/RG.2.2.29245.15846

[70] Spector, B. (2008). "Business Responsibilities in a Divided World": The Cold War Roots of the Corporate Social. Enterprise \& Society, 9(2), 314-336. Retrieved from https://www.jstor.org/stable/pdf/23701370.pdf?casa_token=qKh49dRF8mcAAAAA: LGCtMSm81JYOtLyr8XKUTj091F-vngEc5STzqzPEIRCfWqznwlblee2XUTD1bCV0IHtKXVqC9Z_ _nGOv-ftSzjpVkbPnlL6Z8iQyPbNNWjV7rfkwTaaK7A

[71] Székely, F., \& Knirsch, M. (2005). Responsible Leadership and Corporate Social Responsibility:: Metrics for Sustainable Performance. European Management Journal, 23(6), 628-647. doi:10.1016/j.emj.2005.10.009. doi:doi:10.1016/j.emj.2005.10.009

[72] Van de Velde, E., Vermeir, W., \& Corten, F. (2005). Corporate social responsibility and financial performance. Journal of Business in Society 5(3), 129-138. DOI: 10.1108/14720700510604760.

[73] Velázquez, L. V., \& Vargas-Hernández, J. G. (2012). LA SUSTENTABILIDAD COMO MODELO DE DESARROLLO RESPONSABLE Y COMPETITIVO. Ingeniería de Recursos Naturales y del Ambiente, 97-107.

[74] Venanzi, D. (2013). Stakeholder Ratings and Corporate Financial Performance: Socially Responsible for What? Corporate Ownership and Control 10(4), DOI: 10.2139/ssrn.2188859.

[75] Waddock, S., \& Graves, S. (1997). The Corporate Social Performance-Financial Performance Link. Strategic Management Journal. Vol. 18, No. 4 , 18(4), 303-319. https://www.jstor.org/stable/3088143. 
[76] Wang, H., Tong, L., Takeuchi, R., \& George, G. (2016). Corporate Social Responsibility: An Overview and New Research Directions: Thematic Issue on Corporate Social Responsibility. Academy of Management Journal, 59(2), 534-544. https://doi.org/10.5465/amj.2016.5001.Retrievedfromhttps://ink.library.smu.edu.sg/ lkcsb_research/4942/

[77] Wilson, M. (2003). Corporate sustainability: : What is it and where does it come from? Ivey business journal, $67(6), 1-5$.

[78] Wooldridge, J. (2002). Econometric analysis of cross section and panel data. Cambridge, Massachusetts: MIT Press.

[79] Wooldridge, J. (2010). Econometric Analysis of Cross Section and Panel Data. Cambridge, Massachusetts: MIT Press.

[80] World Commission on Environment and Development. (1987). Our common future. Oxford. (O. U. Press, Ed.) pag.143.

[81] Xiao, Y., Faff, R., Gharghori, P., \& Lee, D. (2013). An Empirical Study of the World Price of Sustainability. Journal of Business Ethics, 114(2), 114:297. https://doi.org/10.1007/s10551-012-1342-2. doi:https://doi.org/10.1007/s10551-012-1342-2

[82] Yu, L. (2014). Performance of Socially Responsible Mutual Funds. Global Journal of Business Research, 6(2), 9-17.

[83] Yustas López , M. (2014). El producto en la tienda: El panel de detallistas en la investigación de mercados. Madrid: UNIVERSIDAD PONTIFICIA COMILLAS. 